\title{
Parameterization and Evaluation of a Spatiotemporal Model of the Potato Late Blight Pathosystem
}

\author{
P. Skelsey, G. J. T. Kessel, W. A. H. Rossing, and W. van der Werf
}

First and fourth authors: Wageningen University, Department of Plant Sciences, Crop and Weed Ecology Group, P.O. Box 430, 6700 AK Wageningen, the Netherlands; second author: Plant Research International, P.O. Box 16, 6700 AA Wageningen, the Netherlands; and third author: Wageningen University, Department of Plant Sciences, Biological Farming Systems Group, P.O. Box 9101, 6700 HB, Wageningen, the Netherlands.

Current address of first author: Evolutionary Genetics Group, University of Groningen, P.O. Box 14, 9750 AA Haren, the Netherlands. Accepted for publication 17 October 2008.

\begin{abstract}
Skelsey, P., Kessel, G. J. T., Rossing, W. A. H., and van der Werf, W. 2009. Parameterization and evaluation of a spatiotemporal model of the potato late blight pathosystem. Phytopathology 99:290-300.

A spatiotemporal, integrodifference equation model of the potato late blight pathosystem is described. Formerly, the model was used in a theoretical context to analyze and predict epidemic dynamics in spatially heterogeneous mixtures of host genotypes. The model has now been modified to reflect a research interest in interactions between genotype, environment, landscape, and management. New parameter values describing host-pathogen interactions were determined and new environment-pathogen relationships included. A new analytical equation describing lesion expansion and associated necrosis has also been developed. These changes prompted a need to assess the quality of model predictions. Cultivar-isolate-specific interactions were characterized in the model using three quantitative components of resistance: infection efficiency, lesion growth rate, and sporulation intensity. These were measured on detached potato leaflets in the laboratory. Results of a sen-

expansion was separated from the epidemic process of lesion propagation providing two reference curves for diagnosing observed epidemics. The spatial component of the model was evaluated graphically in order to determine if realistic rates of focal expansion for potato late blight are produced. In accordance with theory, the radius of a predicted focus increased linearly with time and a constant focal velocity was reached that compared well with published experimental data. Validation data for the temporal model came from 20 late blight epidemics observed in field trials conducted in the Netherlands in 2002 and 2004. The field data and model were compared visually using disease progress curves, and numerically through a comparison of predicted and observed $t_{5}$ and $t_{50}$ points (time in days until 5 and 50\% disease severity is reached, respectively) and relative areas under the disease progress curve values. Temporal model predictions were in close agreement with observational data and the ability of the model to translate measured resistance components, weather data, and initial conditions into realistic disease progress curves without the need for calibration confirms its utility as a tool in the analysis and diagnosis of epidemics.
\end{abstract} sitivity analysis illuminate the effect of different quantitative components of resistance and initial conditions on the shape of disease progress curves. Using the resistance components, the epidemic process of lesion
Additional keywords: convolution, dispersal, fast Fourier transforms, Phytophthora infestans, simulation.
It is well documented that aerial dispersal of sporangia is a crucial process in the development of potato late blight epidemics $(12,13,31)$; however, knowledge of the spatial aspects of spore dispersal is still limited. Management of potato late blight could benefit from prediction of the risk posed to potato fields from external inoculum sources of Phytophthora infestans, but as spore influx depends on a complex interplay of population biological, atmospheric, and spore survival processes, it is difficult to predict. Further questions remain regarding how the composition, configuration, and connectivity of landscapes influence pathogen population dynamics. Optimizing the spatial deployment of host resistance could lead to improvements in disease control, an increase in the durability of resistance gene resources, and a reduction in the chemical requirements of potato cultivation in large growing areas. An enhanced understanding of the spatial aspects of epidemic development at the field- and landscape-scale could therefore lead to the identification of new operational and strategic management practices for potato late blight. Spatiotemporal simulation models will be particularly useful in this

Corresponding author: P. Skelsey; E-mail address: p.skelsey@ rug.nl

doi:10.1094/PHYTO-99-3-0290

(c) 2009 The American Phytopathological Society endeavor as they facilitate a rapid evaluation of disease management practices at scales where experimentation is not always possible.

The model described in this study is a spatiotemporal/integrodifference equation model of the potato late blight pathosystem (P. infestans-Solanum tuberosum). Previously, the model was used to study the influence of different scales and patterns of host genotypes on development of focal and general epidemics (25). In this theoretical context, it was not of prime importance to use parameter values relating to real potato cultivars. As it is the longterm aim of our research to further develop and utilize this model to investigate new strategies for the regional management of potato late blight, some modifications were required. With these objectives in mind, the model was recently updated to include a simple set of climatic relations based on the experimental results of Zwankhuizen and Zadoks (37) and Rotem et al. (24). Parameter values describing cultivar-isolate interactions have also been modified. Three quantitative components of resistance were measured in the laboratory using a sample of potato cultivars and isolates from the Netherlands: the infection efficiency, $1(-)$, the radial lesion growth rate, $\rho\left(\mathrm{m} \mathrm{day}^{-1}\right)$, and the sporulation intensity, $\sigma\left(\# \mathrm{~m}^{-2}\right)$. A new analytical equation for describing lesion expansion has also been incorporated. In light of these recent changes, it is now useful to assess the quality of model predictions in comparison with observed epidemics in the field. 
The primary value of the model is heuristic; i.e., targeted towards increasing our understanding of epidemic dynamics. The model must therefore remain logically tractable and transparent, yet it must produce realistic results that are sufficiently close to real world observations to be considered useful. Thus, our goal in model evaluation is neither to achieve nor force a high level of predictive accuracy per se; evaluation in this context refers to the ability of the model to translate measured resistance components, weather data, and initial conditions into realistic disease progress curves, using as simple and transparent a model as possible, based on the principle of parsimony, also referred to as Ockham's razor $(9,29)$.

Extensions to the model and laboratory experiments used to derive quantitative components of resistance for a number of potato cultivar-isolate combinations are described. A sensitivity analysis is conducted in order to improve understanding about the effect of new model parameter values and initial conditions on the shape of disease progress curves. The spatial component of the model is evaluated by comparing emergent focal expansion velocities with a rate previously determined by Minogue and Fry (20). The quality of temporal model predictions is then tested through comparison with measured epidemics in experimental field plots in the Netherlands. The degree of confirmation of temporal model predictions by observational data is assessed using graphical and numerical methods.

\section{THEORY AND APPROACHES}

An integrodifference equation model for spatial epidemics of $\boldsymbol{P}$. infestans. Integrodifference equations (IDEs) are the spatially explicit equivalent of difference equation models. They are discrete-time, continuous-space models for the growth and spread of biological populations. As noted by Neubert et al. (22), "IDEs contain two components: (i) difference equations, which model growth and interactions during a sedentary stage and (ii) redistribution kernels, which characterize movement during a dispersal stage."

In the model, space is described as a two-dimensional grid, in which each cell represents a potato plant. The Phytophthora population in the model is age- and spatially-structured and internal model accounting keeps track (for every plant in the field) of the number of lesions according to the day of establishment; after $t$ days of simulation there will be $t$ age classes of lesions. Thus, the time step is taken to be 1 day, and not a generation, as is done in some difference equation models. Lesions in the model are circular and grow radially, producing a new ring of growth (annulus) each day, the area of which is calculated as

$$
d A_{t}=2 \pi \rho^{2} t\left(1-\frac{A}{K}\right) d t
$$

where $A_{t}=$ the area $\left(\mathrm{m}^{2}\right)$ of a single lesion of age class $t$ (days), $\rho=$ the radial lesion growth rate $\left(\mathrm{m} \mathrm{day}^{-1}\right), A=$ the total diseased area per plant $\left(\mathrm{m}^{2}\right)$, and $K=$ leaf area of a plant $\left(\mathrm{m}^{2}\right)$. Table 1 provides a summary of symbol definitions and dimensions (all parameters are given in standard SI units). New lesion annuli are latent for a period of time, $\lambda$ (days), set at 5 days for all cultivarisolate interactions. At age $\lambda+1$ day, latent areas become infectious and produce spores. The infectious period is set to 1 day for all cultivar-isolate interactions. During each successive day, as another annulus of daily radial growth reaches age $\lambda+1$ day, this annulus will sporulate. The latent, infectious, and dead lesion areas on each host plant are calculated by summing over age classes the number of lesions in each age class, multiplied by the age-specific area of latent, infectious, and necrotic tissue per lesion. Lesions are aged at a daily time step. Spore production intensity per unit of infectious leaf area is accounted for by the parameter $\sigma\left(\# \mathrm{~m}^{-2}\right)$. All spores that are produced are assumed to be released. Spores are redistributed according to a radial Laplace kernel (25). After sporulation, infectious areas are classed as necrotic. Necrosis of host tissue is also caused by the "girdling" of leaves and stems by lesions. Following van Oijen (32), girdling proceeds at a rate equal to the increase in severity, i.e., for every increase in latent area calculated by equation 1, an equal amount of healthy tissue is classed as necrotic. Leaf lesion coverage and areas of necrotic leaf tissue caused by $P$. infestans are assumed to be distributed homogeneously throughout the canopy. The agestructured population model for lesions on each plant is updated daily, by "aging" the existing lesions by 1 day, and starting new lesions at a rate calculated as the product of the number of landed spores per square meter of leaf area in a day, the infection efficiency, $1(-)$, and the fraction of area not yet occupied by colonies of $P$. infestans (including latent, infectious, and necrotic tissue). This fraction is also used to limit real expansion of lesions (due to competition for space at the plant level).

Solution of spatial phenomena. As described in Skelsey et al. (25), spatial phenomena in the model are solved by performing convolutions between spatial distributions of spores and dispersal kernels. Convolutions are implemented in the model via fast Fourier transforms; a technique that greatly enhances the speed and accuracy of the numerical solution of the model. Spatial distributions are transformed into the Fourier domain, multiplied, and the result is back-transformed into the spatial domain. This solution method requires periodic boundary conditions, therefore Fourier domain arrays are duplicated an infinite number of times to the left, right, top, and bottom. This means that each edge of the array is connected to the opposite edge of an identical array. This leads to "wrap-around" effects, whereby any spore dispersing outside of the field's borders will "reappear" on the opposite edge of the field when spatial distributions are backtransformed into the spatial domain. This artifact of the solution methodology can be used to provide a representation of interplot interference (spore exchange between neighboring plots), which is based on the spatial characteristics of the experimental site. Prior to convolution, spatial distributions are "padded" with a border of "non-crop" area (zero-padding) that matches the spacing between experimental plots. Spores that are redistributed by the kernel into the non-crop area are lost from the system and do not contribute to epidemic development. Spores that are redistributed beyond the border of non-crop area (i.e., further than the spacing between plots) reappear on the opposite edge of the source plot and are assumed to come from a neighboring donor plot. The extent of zero-padding can also be increased to completely mitigate wrap-around effects. This is useful in the simulation of completely isolated plots or fields.

Environment-pathogen interactions. The model has a daily time step, which is suitable for a study of epidemic dynamics over the length of an entire growing season, but inappropriate for the incorporation of environment-pathogen interactions. Some meteorological variables that have profound effects on the pathogen, such as relative humidity, can show a marked variation over a $24 \mathrm{~h}$ period and therefore it is not possible to average these variables over a day and use them in any meaningful analysis of epidemic dynamics. In the model a simplifying assumption is made that colony growth continues within the leaf tissue and sporangia are produced, dispersed, and deposited irrespective of the weather, however, successful germination of dispersed sporangia is weather dependent. Two simple rules are used to analyze hourly weather data and determine if the conditions during a $24 \mathrm{~h}$ period (1600 to $1600 \mathrm{~h}$; one time step in the model) are suitable for sporangia to cause infection. The first rule determines if the conditions each hour are suitable for the germination process to take place. Following Zwankhuizen and Zadoks (37), an "infection-hour" is defined as any hour where the temperature is between 10 and $27^{\circ} \mathrm{C}$ and the relative humidity is greater than $90 \%$. The second rule determines the number of consecutive 
infection hours required to allow germination to reach completion in a $24 \mathrm{~h}$ period. This rule is based on the average temperature during consecutive infection-hours, $T_{\text {inf }}\left({ }^{\circ} \mathrm{C}\right)$, and the experimental results of Rotem et al. (24): for $10^{\circ} \leq T_{\text {inf }} \leq 15^{\circ}, 12$ consecutive infection-hours are required; for $15^{\circ}<T_{\text {inf }} \leq 20^{\circ}, 18$ consecutive infection-hours are required; for $20^{\circ}<T_{\text {inf }} \leq 27^{\circ}, 24$ consecutive infection-hours are required; and for $T_{\mathrm{inf}}<10^{\circ}$ and $T_{\mathrm{inf}}>27^{\circ}$, penetration does not occur. If the conditions of these rules are not met, then infection does not take place and the parameter 1 (infection efficiency, -) is set to 0 for that $24 \mathrm{~h}$ period. These rules therefore act as a simple "switch"; the polycyclic component of the epidemic is allowed to proceed under suitable conditions and it is "switched off" under adverse conditions.

Model parameterization-pathogenicity experiments. Unsprayed, fully grown new leaves from the Wageningen field experiments (described later) were sampled in 2002, 2003, and 2004 and taken to the laboratory to determine $1, \rho$, and $\sigma$. Five potato cultivars with different levels of resistance against $P$. infestans were used: Agria (5.5), Aziza (7.5), Bintje (3), Remarka (6.5), and Sante (4.5). Foliar resistance ratings to potato late blight according to the Dutch National variety list are given in brackets (on a scale of $1=$ very susceptible to $9=$ very resistant). Two different $P$. infestans isolates (IPO82001 and IPO428-2) were used as inoculants in both the pathogenicity studies and the field experiments. Isolates and their key characteristics, as determined by Flier and Turkensteen (8), are listed in Table 2.

Culturing and preparation of inoculum. $P$. infestans isolates IPO82001 and IPO428-2 were stored in liquid nitrogen as sporangial suspensions in a $15 \%$ dimethylsulphoxide solution. Isolates taken from long-term storage were first cultured on tuber

TABLE 1. Symbols used in this study

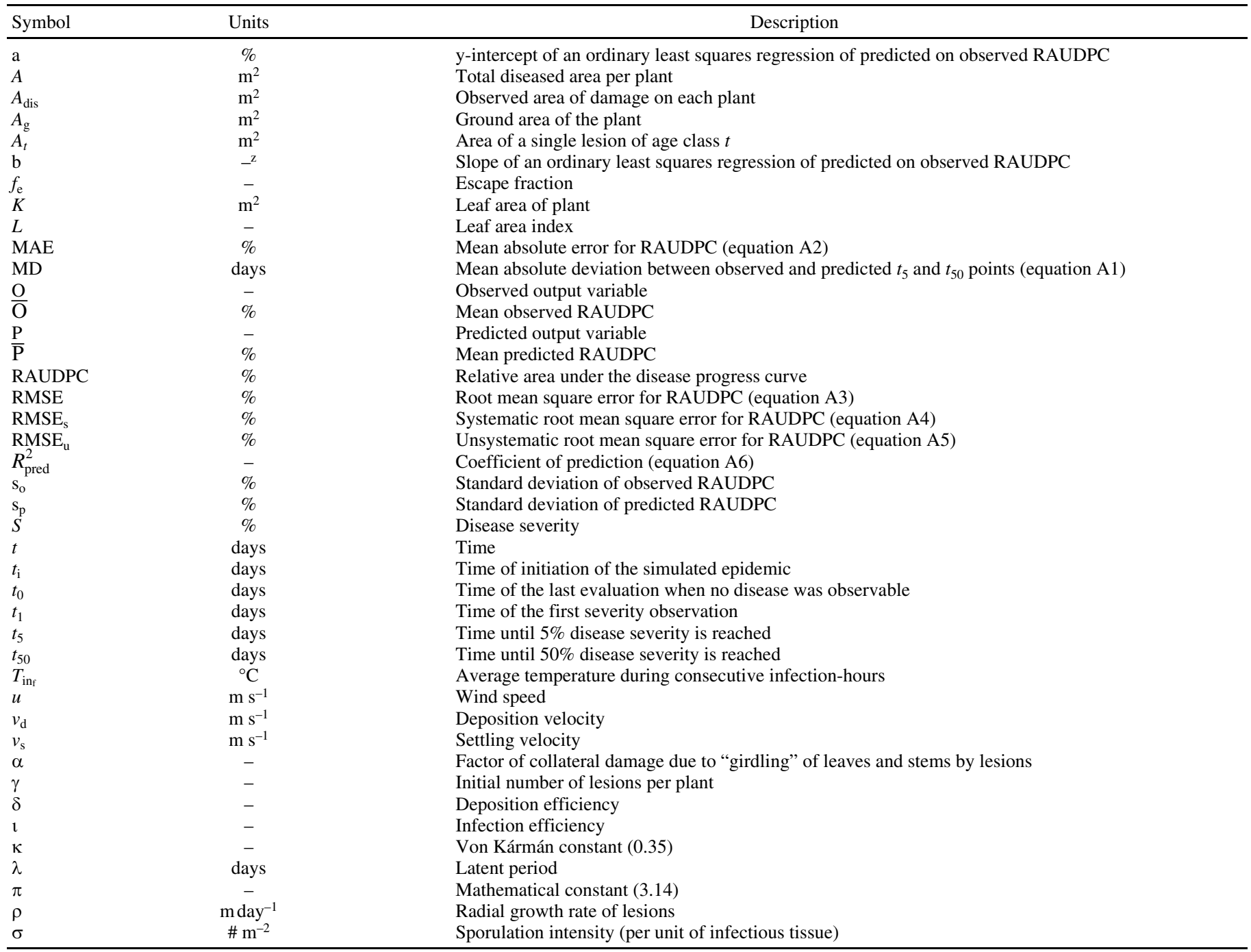

$\mathrm{z}$ - Signifies that the defined quantity is dimensionless.

TABLE 2. Isolates of Phytophthora infestans used to determine cultivar specific resistance components and to inoculate the field experiments used for model validation

\begin{tabular}{lccccc}
\hline Isolate & Year of collection & Location & Race $^{\mathrm{x}}$ & Mating type & mtDNA haplotype \\
\hline IPO82001 & 1982 & Gembloux $\left(\mathrm{B}^{\mathrm{y}}\right)$ & 1.2 .3 .4 .5 .6 .7 .10 .11 & $\mathrm{~A} 2$ & Ia \\
IPO428-2 & 1992 & Ede $\left(\mathrm{NL}^{z}\right)$ & 1.2 .3 .4 .5 .6 .7 .8 .9 .10 .11 & A2 & Ia
\end{tabular}

${ }^{\mathrm{x}}$ Determined by Flier and Turkensteen (8) using the R-gene differential set of potato clones for race identification: r0 (Bintje), R1, R2, R3, R4, R5, R6, R7, R8, R9, R10, and R11 $(3,18)$.

y Belgium.

${ }^{\mathrm{z}}$ Netherlands. 
slices of the general susceptible potato cultivar Bintje by incubation in the dark at $15^{\circ} \mathrm{C}$ for 5 to 7 days. When sporulating mycelium was present, small pieces of mycelium were placed on the abaxial (lower) epidermis of leaflets of cultivar Bintje, which were placed with the abaxial side up in $9 \mathrm{~cm}$ petri dishes containing $10 \mathrm{ml}$ of $2 \%$ water agar. Inoculated leaflets were kept in a climate chamber at $15^{\circ} \mathrm{C}$ with a $16 \mathrm{~h}$ light period. Leaflets densely covered with sporulating mycelium were obtained after 7 days of incubation. Sporangial inoculum was prepared by washing these leaves in $20 \mathrm{ml}$ of tap water. The concentration was adjusted to $1 \times 10^{4}$ or $1 \times 10^{5}$ sporangia $\mathrm{ml}^{-1}$ with a Coulter Counter Z1 (Coulter Electronics Inc.) and kept at $18^{\circ} \mathrm{C}$. The suspensions were used as inoculum within 30 min after preparation.

Plant material. Potato cultivars Agria, Aziza, Bintje, Remarka, and Sante were grown in $3.75 \times 3.75 \mathrm{~m}$ plots under field conditions during the spring of 2002, 2003, and 2004 in Wageningen, the Netherlands. Leaf material not protected with fungicides was sampled from the fourth and fifth leaf layer from the top when the plants were 7 to 9 weeks old.

Quantification of resistance components. Resistance components $l, \rho$, and $\sigma$ were quantified in a series of bioassays. Lateral leaflets were placed in $9 \mathrm{~cm}$ petri dishes containing $10 \mathrm{ml}$ of $2 \%$ water agar, one leaflet per petri dish, lower (abaxial) side up. To determine 1 , ten $10-\mu$ droplets of a sporangial suspension containing $1 \times 10^{4}$ sporangia $\mathrm{ml}^{-1}$ were placed on the abaxial side of each leaflet. Petri dishes with the inoculated leaf disks were placed in plastic trays, which were then enclosed in a transparent polythene bag to avoid desiccation. The trays were placed in the dark in a climate chamber at $15^{\circ} \mathrm{C}$. After $24 \mathrm{~h}$, incubation was continued at $15^{\circ} \mathrm{C}$ and $16 \mathrm{~h}$ photoperiod. The number of successful inoculations (spreading lesions) per leaflet was determined after 7 days incubation and 1 was calculated according to Swallow (28). To determine $\rho$ and $\sigma$ one 10- $\mu$ l droplet of a sporangial suspension containing $5 \times 10^{4}$ sporangia $\mathrm{ml}^{-1}$ was placed on the abaxial side of each leaflet. Petri dishes containing inoculated leaf disks were placed in plastic trays, which were then enclosed in a transparent polythene bag to avoid desiccation. The lesion diameter was measured along two perpendicular axes at least twice before the lesion reached the edge of the leaflet, and $\rho$ was calculated from these measurements. Following the last measurement, the sporangia were released from the lesions by shaking the leaflet in $10 \mathrm{ml}$ of Isoton II electrolytic buffer (Coulter Electronics Inc.) with a vortex mixer. After dilution, the sporangial concentration was measured in three $0.5-\mathrm{ml}$ subsamples using a Coulter counter and $\sigma$ was calculated from the lesion surface area and the number of sporangia produced on the lesion.

Statistical analysis. Data came from seven laboratory experiments (described previously): two experiments in 2002, three experiments in 2003 and two experiments in 2004. Within the separate experiments, each petri dish containing one potato leaflet was considered a replicate. Four replicate leaflets per experiment were used to determine $\imath$ and $\rho$ in 2002 and 2004. Five replicate leaflets per experiment were used to determine 1 and $\rho$ in 2003. Two replicate leaflets were used to determine $\sigma$ in all seven experiments. Linear mixed modeling (LMM) was used to test for possible interactions between year and experiment, year and cultivar, cultivar and inoculum, and between year and inoculum, using the statistical package Genstat for Windows 9th edition (VSN International Ltd., Hemel Hempstead, 2007), extended with the Biometris procedure library (11).

No significant interactions were present between year and cultivar, and between year and inoculum, therefore the LMM was fitted with fixed factor cultivar $\times$ inoculum and random factor year $\times$ experiment (treating the experiments of 3 years as seven independent experiments). The data set was unbalanced and therefore the LMM was estimated using residual maximum likelihood (REML). A normal distribution was assumed for $\imath$ and $\rho$ and analysis of residuals supported this assumption. Some residual plots showed a small number of values reaching a lower limit (0) or an upper limit. Residuals showed a significant departure from a normal distribution for $\sigma$; therefore, a Poisson distribution was assumed and $\sigma$ was analyzed using a generalized linear mixed model (GLMM).

Sensitivity analysis. In order to gain insight into possible causal effects for any differences between observed and predicted epidemics, the influence of individual resistance components, the parameter deposition efficiency and initial inoculum level on the shape of disease progress curves were studied. Environmentpathogen interactions were not simulated so that the influence of the parameter of interest on model results was separated from the influence of the weather. In order that this assumption did not result in rapid epidemic development, which could obscure subtle changes in disease progress curves arising from model manipulations, parameters defining a resistant host-pathogen interaction were selected for analysis. Measured parameter values for cultivar Aziza and isolate IPO428-2 (Results section), together with assumed standard values of $\lambda=5$ days (latent period) and $\delta=1$ (deposition efficiency, -), and an initial severity level, $\gamma$, of 10 lesions per plant provided the values for the "standard" epidemic. Additional necrosis caused by girdling of leaves and stems by lesions was not simulated. Plot size was fixed at $3.75 \times 3.75 \mathrm{~m}$ in accordance with the field data used to validate temporal model predictions (see field experiments section later). The simulated plot was padded with a large border of non-crop area (isolated plot) in order to facilitate net loss of spores from the spatial domain (see solution of spatial phenomena section). Parameters $\delta$, $\gamma, \imath, \rho$, and $\sigma$ were varied in turn over a range of $-/+$ one order of magnitude, whereas $\lambda$ ranged between 2 and 7 days.

In some plant pathosystems, e.g., Puccinia spp. on many hosts, the pathogen causes discrete lesions that remain constricted in size over the lifespan of the host. In contrast, in potato late blight, lesions continue to expand after their initial appearance, thus, potato late blight epidemics can be abstracted to a combination of two processes: lesion expansion and the polycyclic process of lesion propagation. A further set of analyses was conducted whereby the relative importance of these two epidemic processes was demonstrated through adjustment of the value of resistance components. Parameters defining the less resistant host-pathogen interaction of cultivar Remarka and isolate IPO82001 (Results section) were selected for analysis, with assumed standard values of $\lambda=5$ days (latent period) and $\delta=1$ (deposition efficiency, - ). Epidemics were initiated with an initial severity level, $\gamma$, of 10 lesions per plant. Additional necrosis caused by girdling of leaves and stems by lesions was not simulated. Complete dominance of the lesion expansion process was obtained by setting $\delta, \imath$, or $\sigma$ to zero. Dominance of the polycyclic process was obtained by reducing $\rho$ and increasing $\sigma$ by three orders of magnitude, respectively. Plot size was again fixed at $3.75 \times 3.75 \mathrm{~m}$ and padded with a large border of non-crop area (isolated plot).

Evaluation of the spatial component of the model. The twodimensional radial Laplace kernel used in the model is parameterized for the dispersal of $P$. infestans spores under nonirrigated conditions (23). It is of interest to confirm if the kernel and the model can produce realistic rates of focal expansion for potato late blight. Such data were not collected as part of this study but a spread velocity of $3.0 \mathrm{~m} \mathrm{day}^{-1}$ was determined experimentally by Minogue and Fry (20) for potato late blight for a susceptible cultivar not protected with fungicides. In order to replicate these field experiments exactly, the model requires cultivar-isolatespecific parameters and site-specific weather data, and these are unknown. However, as the intention of this comparison is simply to evaluate if predicted focal expansion rates lie within the realm of biological plausibility, we proceeded using parameter values for cultivar Bintje and isolate IPO82001 (Results section).

An epidemic was simulated in a 1 ha field via inoculation of the center plant with 1 lesion. The field was padded with a large 
border of non-crop area (isolated field) in order to facilitate net loss of spores from the spatial domain (solution of spatial phenomena section). No weather data were available so perfect weather conditions for disease were assumed. Focal radius was derived at each time step by first calculating the crop area infected. The area of the focus was determined by counting the number of plants with a disease severity greater than $1 \%$, and multiplying this number by the ground area occupied by a plant. Focal radius was calculated through manipulation of the formula for the area of a circle, and the focal expansion rate determined through linear regression of focal radius on time. Radius calculations prior to day 15 were ignored in order to avoid distortion of the result created by the initial phase of focus build up.

Evaluation of temporal model predictions-field experiments. Observational data used to validate temporal model predictions came from field trials conducted in the Dutch location of Wageningen in 2002 and 2004 (Table 3). The two different inocula used in the pathogenicity studies described previously (Table 2) were each assigned to a separate area of the experimental site. The two areas were separated by $5 \mathrm{~m}$ of bare soil, $5 \mathrm{~m}$ of maize and another $5 \mathrm{~m}$ of bare soil. Each area contained a randomized block experiment with the appropriate cultivars in three replications. Individual plots within the randomized block experiments measured $3.75 \times 3.75 \mathrm{~m}$ and were separated by $3 \mathrm{~m}$ of bare soil. Plots were spray inoculated (on a wet crop) with $300 \mathrm{ml}$ of $P$. infestans sporangial suspension $\left(1.0 \times 10^{4}\right.$ sporangia $\mathrm{ml}^{-1}$ ) just before dark on 24 June 2002 and 7 August 2004. Disease severity was assessed twice per week using a percentage scale adapted from James (14). Experimental plots were desiccated after 35 days in 2002, and 29 days in 2004. Temperature and relative humidity measurements for the 2002 field trials came from the nearby $(<1 \mathrm{~km})$ Wageningen University weather station (Haarweg). During the 2004 field trials, relative humidity and temperature were monitored using an electronic sensor (Pow 835VDG, Rotronic AG, Bassersdorf, Switzerland) positioned within the canopy at $0.35 \mathrm{~m}$ above ground level. Data were recorded every 15 min by a data logger (Delta-T Devices Ltd., Cambridge, UK).

Estimation of initial inoculum. Lesion count data were absent from the field data, therefore it was necessary to develop a scheme with which to estimate the initial number of lesions for the simulations, based upon severity observations. It was assumed that late blight first became observable between the last field evaluation on which no lesions were observed and the first observed severity measurement. The method of Andrade-Piedra et al. (2) was followed to estimate the time of initiation, $t_{\mathrm{i}}$ (days), of the simulated epidemic:

$$
t_{\mathrm{i}}=\left[\left(t_{0}+t_{1}\right) / 2\right]-\lambda
$$

where $t_{0}=$ the day of the last evaluation when no disease was observable, and $t_{1}=$ the day of the first severity observation. The observed area of damage on each plant, $A_{\text {dis }}\left(\mathrm{m}^{2}\right)$ at $t_{1}$ was given by:

$$
A_{\text {dis }}=L_{t_{1}} A_{\mathrm{g}} S_{t_{1}} / 100
$$

where $L=$ leaf area index $(-), A_{\mathrm{g}}=$ the ground area of the plant $\left(\mathrm{m}^{2}\right)$, calculated on the basis of planting patterns in the Nether- lands as the product of the distance between plants and the distance between rows $(0.3 \times 0.75 \mathrm{~m})$, and $S=$ severity $(\%)$. In order to calculate the area of damage caused by a single lesion that had been growing since $t_{\mathrm{i}}$, equation 1 was rewritten to include the additional necrotic area caused by girdling of leaves and stems by a lesion:

$$
\frac{d A_{t}}{d t}=\alpha 2 \pi \rho^{2} t\left(1-\frac{A}{K}\right)
$$

where $t=$ the growing time of the lesion $\left(t_{1}-t_{\mathrm{i}}\right.$, days), and $\alpha=\mathrm{a}$ constant (-) used to describe the factor of additional necrotic area (set to 2 as described previously). Integrating with respect to time provided an analytical equation describing the monocyclic growth process of lesion expansion and associated necrosis caused by girdling of stems and leaves:

$$
A_{t}=K\left[1-\exp \left(-\alpha \pi \rho^{2} t^{2} / K\right)\right]
$$

The initial number of lesions, $\gamma(-)$, applied to each plant in the simulated epidemics was then calculated as the quotient between the diseased area of a plant and the damage caused by a single lesion:

$$
\gamma=A_{\text {dis }} / A_{t}
$$

It is of fundamental importance to obtain an accurate measurement of initial disease severity when observational data are to be used for model validation. Initial severity measurements were low $(\ll 1 \%)$ in all the observed epidemics, and at such low levels the possibility for human error in the visual assessment of disease cannot be ignored. The model was sensitive to initial conditions, and in approximately half of the simulated epidemics, model predictions were improved by using a (larger) severity observation at a later date than $t_{1}$ to calculate $\gamma$. This meant that in certain cases, very small, initial severity estimates were assumed to be subject to human error and were set to zero: in epidemics 3 , 7,8 , and 12 (Table 3), severity was assumed to be $0 \%$ for all observed values where $S \leq 0.001 \%$; in epidemics 4 to 6,14 , and 15 , severity was assumed to be $0 \%$ for all observed values where $S \leq 0.01 \%$; in epidemic 2 , severity was assumed to be $0 \%$ for all observed values where $S \leq 1 \%$; and in epidemics 1 and 16 , severity was assumed to be $0 \%$ for all observed values where $S \leq$ $2 \%$.

Statistical analysis. The quality of temporal model predictions is first assessed through a graphical comparison of disease progress curves and a numerical comparison of $t_{5}$ and $t_{50}$ points (time at which 5 and $50 \%$ severity was reached, respectively). Linear interpolation is used to determine observed and predicted $t_{5}$ and $t_{50}$ points for each epidemic, then the mean absolute deviation, MD (days), between predicted and observed values is calculated. The performance criterion to consider the model valid for correctly predicting the shape of observed disease progress curves is that the mean $t_{5}$ and $t_{50}$ deviations (MD) should not exceed 3 days (approximately $10 \%$ of the duration of observed epidemics).

Observed and predicted relative area under the disease progress curve (RAUDPC) (\%) values are also calculated (4) for each

TABLE 3. Experiments used to validate the potato late blight model

\begin{tabular}{lllll}
\hline Year & Location & $\mathrm{n}^{\mathrm{x}}$ & \multicolumn{1}{c}{ Cultivar $^{\mathrm{y}}$} & Isolate $^{\mathrm{z}}$ \\
\hline 2002 & Wageningen & 3 & Agria (1), Aziza (2), Bintje (3), Remarka (4), Sante (5) & IPO428-2 \\
& & 3 & Agria (6), Aziza (7), Bintje (8), Remarka (9), Sante (10) & IPO82001 \\
2004 & Wageningen & 3 & Agria (11), Aziza (12), Bintje (13), Remarka (14), Sante (15) & IPO428-2 \\
& & 3 & Agria (16), Aziza (17), Bintje (18), Remarka (19), Sante (20) & IPO82001 \\
\hline
\end{tabular}

\footnotetext{
${ }^{x}$ Number of replicates.

${ }^{y}$ The number in parenthesis identifies the epidemic. The same code is used in Figure 4. Foliar resistance ratings according to the Dutch National variety list (scale of $1=$ very susceptible to $9=$ very resistant): Agria (5.5), Aziza (7.5), Bintje (3), Remarka (6.5), and Sante (4.5).

${ }^{z}$ Table 2 provides a description of the isolates.
} 
epidemic and according to the criteria of Willmott $(34,35)$, a complement of summary and difference statistics are used to evaluate model performance: the mean absolute error (MAE) (\%), the root mean square error, RMSE (\%), the systematic root mean square error $\left(\mathrm{RMSE}_{\mathrm{s}}\right)(\%)$, the unsystematic root mean square error $\left(\mathrm{RMSE}_{\mathrm{u}}\right)(\%)$, and Turchin's coefficient of prediction, $R_{\text {pred }}^{2}(-)$, which is a relative difference measure (29). Turchin considers an ecological model to be "successful" if a value between 0.4 and 0.9 is attained for the coefficient of prediction and this is used as a second performance criterion. All statistical formulae are described in the appendix.

Simulation. Observational plots were separated by $3 \mathrm{~m}$ of bare soil on all sides therefore simulated plots used to assess the quality of temporal model predictions are padded on all sides with a 3-m border of non-crop area. Length and width of each compartment cell in the model is set at $0.75 \mathrm{~m}$ (distance between rows) and $0.3 \mathrm{~m}$ (distance between plants) in accordance with these data. The foliage in all experimental plots was fully developed, and epidemics progressed rapidly, therefore host growth is not modeled and leaf area index, $L$, is fixed at 5 in all simulations. Further simplifying assumptions are made with regard to the disease cycle. Under field conditions, with a sparse, open canopy, and vigorous ventilation, a fraction of spores may escape the canopy and contribute to long distance dispersal. At the spatial scale of a micro-plot, these spores would be considered as "lost" from the system. Escape fractions are ignored in this study for two reasons. In the first instance the foliage in all observational plots was fully developed, which would reduce escape considerably. Secondly, vigorous ventilation of potato canopies was unlikely as the experimental site was surrounded on all sides by a tall, thick border of maize, and by a row of mature trees on two sides. In order to verify if this assumption was justified, an escape fraction, $f_{\mathrm{e}}(-)$, was calculated using the mechanistic model for spore escape developed by de Jong et al. (5):

$$
f_{\mathrm{e}}=\exp \left[-L \sqrt{v_{\mathrm{d}} /(\kappa u)}\right]
$$

where $v_{\mathrm{d}}=$ deposition velocity $\left(\mathrm{m} \mathrm{s}^{-1}\right), \kappa=$ the von Kármán constant $(-)$, and $u=$ wind speed $\left(\mathrm{m} \mathrm{s}^{-1}\right)$. Deposition velocity is calculated as $(1,7)$ :

$$
v_{\mathrm{d}}=(1+L) v_{\mathrm{s}}
$$

where $v_{\mathrm{s}}=$ settling velocity $\left(\mathrm{m} \mathrm{s}^{-1}\right)$, given by Gregory (10) as $0.0085 \mathrm{~m} \mathrm{~s}^{-1}$ for P. infestans sporangia. Wind speeds were not measured within the experimental site but data from the nearby Wageningen University weather station were available (Haarweg). Over the course of the 2004 field experiments, wind speeds averaged $2 \mathrm{~m} \mathrm{~s}^{-1}$ at $2 \mathrm{~m}$ height. Assuming a wind speed of $1 \mathrm{~m} \mathrm{~s}^{-1}$ within the sheltered experimental site, and a conservative estimate that wind speeds were half that again within each canopy at half crop height, equations 7 and 8 give an escape fraction of $2 \%$. This low value suggests that the assumption of no spore escape from the canopy is a useful simplification for these data. Continuing with the premise that wind speeds within the experimental site were low, and given that canopies were fully developed, a further simplifying assumption is made that all spores that disperse to another plant deposit on host tissue $(\delta=1)$.

\section{RESULTS}

Pathogenicity experiments. The interactions between cultivar and isolates were statistically significant for $\mathrm{l}(P<0.001), \rho(P<$ $0.001)$, and $\sigma(P<0.05)$. Results for mean resistance component values and significance groupings are given in Table 4 .

Sensitivity analysis. Of the six parameters investigated $(\delta, \gamma, \mathrm{l}$, $\lambda, \rho$, and $\sigma$ ), only four have a fundamentally different effect on the shape of disease progress curves (Fig. 1). The effect of manipulation of $\delta, \mathfrak{l}$, or $\sigma$ on the relative growth rate of epidemics was identical. The same increase in $\delta$, 1 , or $\sigma$ resulted in an identical reduction in the length of the lag period or exponential phase (defined here as the time till 5\% disease severity), and an identical increase in the steepness of the disease progress curve. This was expected as all three parameters exert an influence on the number of new lesions formed in the next generation; $\sigma$ affects the number of spores that are produced and hence arrive at a new site, $\delta$ affects the number that deposit on leaf tissue, and 1 affects the fraction of deposited spores that germinate. In terms of their effect on the shape of disease progress curves, these three parameters can thus be grouped together into a single "propa-

TABLE 4. Mean infection efficiency ( $(1)$, radial lesion growth rate $(\rho)$, and sporulation intensity $(\sigma)$ of five potato cultivars inoculated with two Phytophthora infestans isolates from the Plant Research International collection in the Netherlands ${ }^{v}$

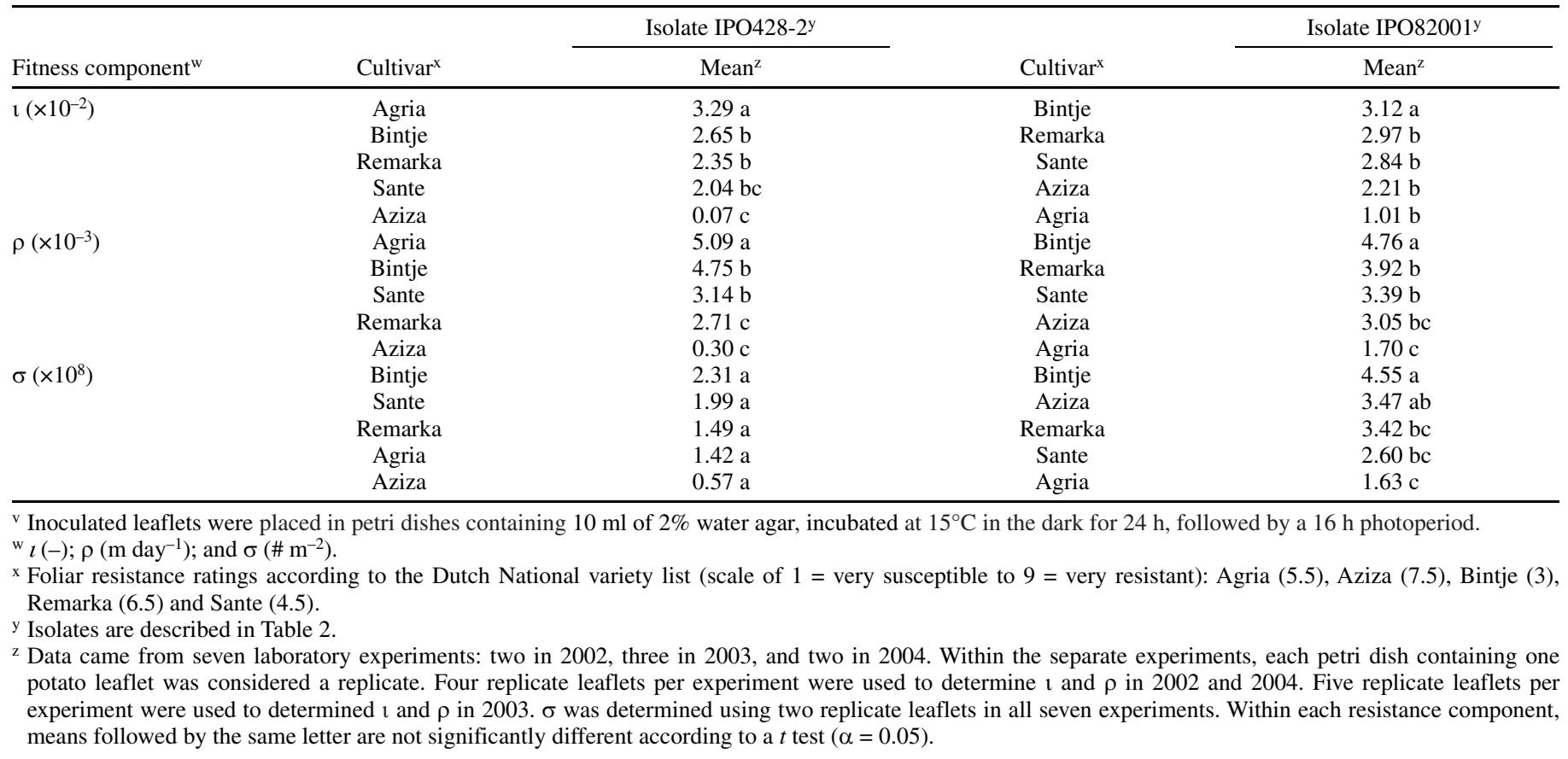


gation parameter," where the aggregate effect depends not so much on the value of each single parameter as on their product.

Manipulation of $\rho$ resulted in the largest deviations from the standard curve (Fig. 1). Changes in the value of this parameter had a major effect on the length of the lag period and the steepness of the disease progress curve. This was also expected since $\rho$ represents the radial growth rate of lesions and therefore affects the areal growth rate quadratically (33). Moreover, the value of $\rho$ also affects the rate of production of new lesions.

The latency period, $\lambda$, also plays a role in the polycyclic process but its effect on disease progress curves was minimal in comparison with the other parameters tested (Fig. 1). As $\lambda$ was increased, the lag period lengthened and the steepness of the curve reduced very slightly. This was expected as when $\lambda$ is longer, the exponential growth rate of an epidemic decreases (30). The reason $\lambda$ had a seemingly minimal effect in comparison to the propagation parameters $\delta, \mathrm{l}$, and $\sigma$, is because it was varied over a narrow, but realistic range; $\lambda$ would have to be very short for a change in the order of 1 or 2 days to have a significant effect on the rate of epidemic development. Furthermore, lesion expansion (as determined by $\rho$ ) gives rise to new inoculum in a shorter time than the cycling through latent periods (16).
The effect of $\gamma$ on the shape of the disease progress curve was slightly different from the other parameters (Fig. 1). Lesions act independently of one another in the exponential phase of an epidemic; thus an increase in the initial number of lesions did not alter the relative growth rate of the epidemic. The shape of the disease progress curve was therefore unaltered; the increased lesion input only quickened the onset of the epidemic and shifted the disease progress curve to the left on the time-axis.

Two extreme examples of a disease progress curve are illustrated in Figure 2. The first curve represents a Phytophthora strain with no local spore production. It is assumed that the initial inoculum came from an external source and that the polycyclic process of reproduction and establishment of new lesions is effectively shut off. This was achieved by setting either $\delta$, 1 , or $\sigma$ to zero. Thus, the pure consequences of lesion expansion are realized, resulting in a gentle s-shaped curve with a short lag period that is very rounded in the terminal phase. Alternatively, for a Phytophthora strain that has very little lesion expansion, the polycyclic process-involving reproduction and establishment of new lesions-dominates the epidemic progress curve, as for a rust disease. To produce the second curve, the contribution of lesion expansion was attenuated by reducing $\rho$ to a minimal amount, and

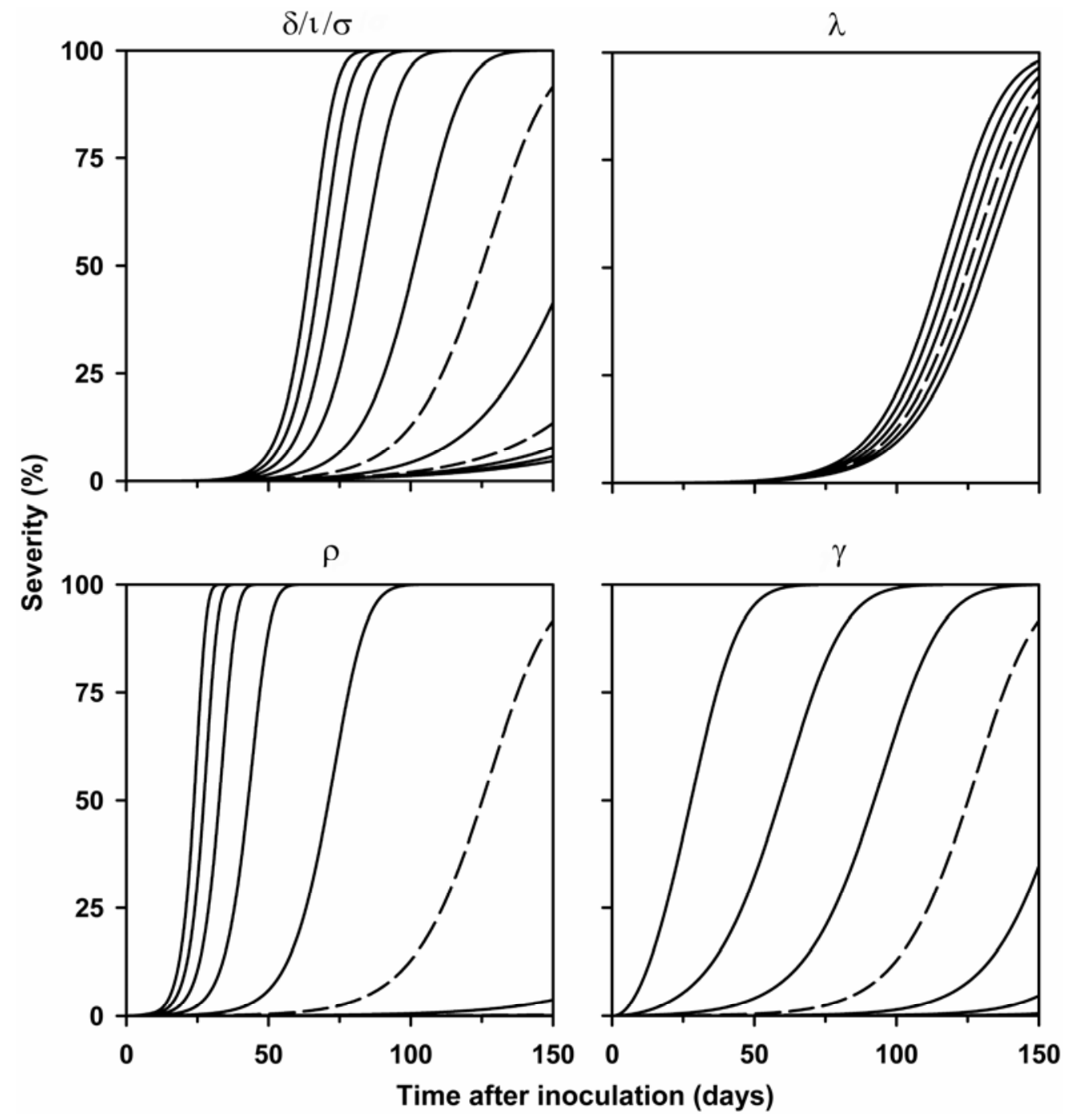

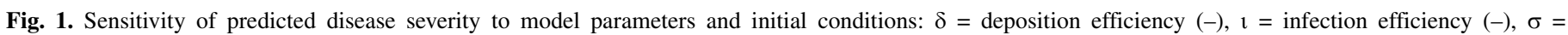

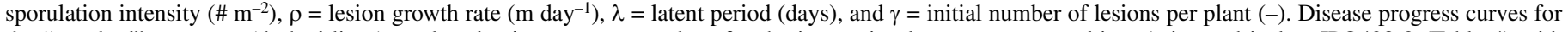

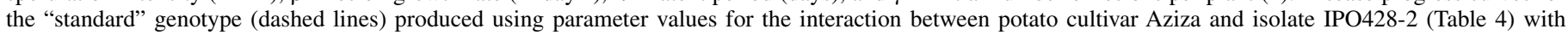

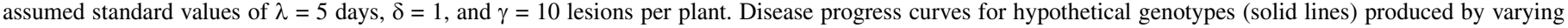

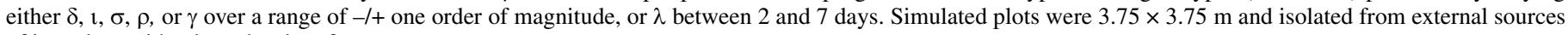
of inoculum with a large border of non-crop area. 
dominance of the polycyclic process increased by boosting sporulation via the parameter $\sigma$. Thus, the epidemic was driven by the formation of new lesions as opposed to the growth of existing lesions. Lesion expansion could not be switched off completely due to the model assumption that lesions have no area immediately after completion of the infection process. This near exponential process, plotted on a linear scale, yields a curve with a long lag period followed by a sudden "explosion" towards $100 \%$ infection. These two curve types can be thought of as the extreme end points of the range of shapes of disease progress curve that can be observed in nature.

Evaluation of the spatial component of the model. Focal radius increased linearly with time and focal velocity reached a constant value of $2.3 \mathrm{~m} \mathrm{day}^{-1}$ (Fig. 3). This compares well with the velocity of $3.0 \mathrm{~m}$ day $^{-1}$ determined experimentally by Minogue and Fry (20), indicating that the spatial component of the model is operating within the bounds of reality.

Evaluation of temporal model predictions. Based on a visual assessment, the disease progress curves generated by the model were a reasonably accurate fit of the observed epidemics in the field (Fig. 4). Observed and predicted $t_{5}$ and $t_{50}$ points, and relative area under disease progress curve (RAUDPC) are presented in Table 5. The mean (absolute) difference, MD, between observed and predicted $t_{5}$ points was 2.3 days with a slightly higher mean difference of 2.7 days for observed and predicted $t_{50}$ points (Table 6). Both these values were within the predefined threshold criterion of 3 days, therefore the model is judged to be valid in terms of correctly predicting the timing of observed disease progress. Further examination of summary measures reveals that there is no significant bias in model predictions $(\overline{\mathrm{P}} \approx \overline{\mathrm{O}})$, and that the model is able to predict the variability contained in the observations $\left(\mathrm{s}_{\mathrm{p}} \approx \mathrm{s}_{\mathrm{o}}\right)$. $\mathrm{RMSE}_{\mathrm{u}}$ is close in value to RMSE, and the proportion of RMSE that can be attributed to systematic errors $\left(\mathrm{RMSE}_{\mathrm{s}}{ }^{2} / \mathrm{RMSE}^{2}\right)$ is very low at 0.01 , confirming that the model is able to predict most of the major trends and patterns in observed RAUDPC. A value of 0.6 was obtained for Turchin's coefficient of prediction, $R_{\text {pred }}^{2}$ (Table 6). This fell within the

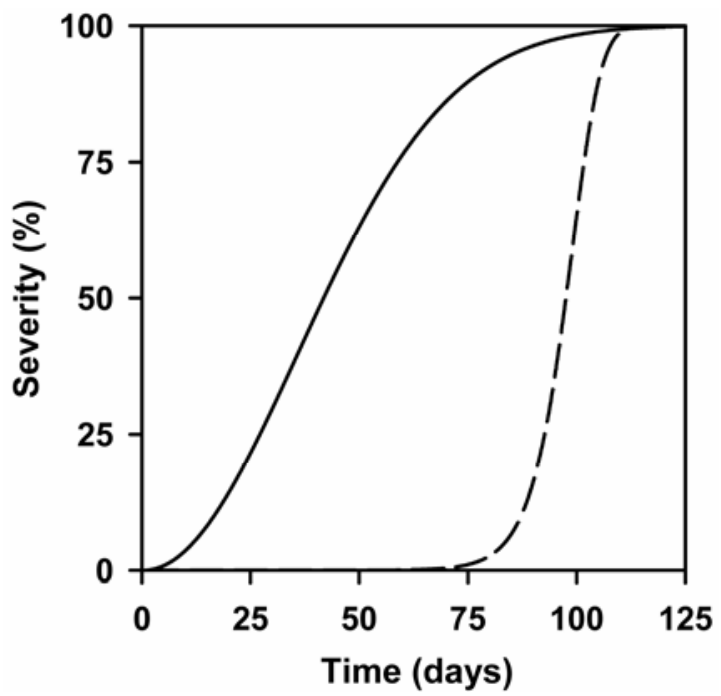

Fig. 2. Division in the effect of resistance components on disease progress curves of potato late blight epidemics. Simulated disease progress curves were obtained by manipulating measured parameter values for the interaction between potato cultivar Remarka and isolate IPO82001 (Table 4). Complete dominance of the lesion expansion process (solid line) is obtained by setting deposition efficiency $(\delta,-)$, infection efficiency $(1,-)$, or sporulation intensity $\left(\sigma, \# \mathrm{~m}^{-2}\right)$ to zero. Dominance of the polycyclic process of lesion propagation (dashed line) is obtained by reducing radial lesion growth rate, $\rho\left(\mathrm{m} \mathrm{day}^{-1}\right)$, and increasing $\sigma$ by three orders of magnitude respectively. Simulated plots were $3.75 \times 3.75 \mathrm{~m}$ and isolated from external sources of inoculum with a large border of non-crop area. Epidemics were initiated with 10 lesions per plant. range defined as a second performance criterion $\left(0.4 \leq R_{\text {pred }}^{2} \leq\right.$ $0.9)$, therefore overall model performance was judged to be acceptable.

\section{DISCUSSION}

A spatiotemporal model of the potato late blight pathosystem was updated with cultivar-isolate-specific parameters, environment-pathogen relations, and a new analytical equation describing lesion expansion and associated necrosis. These modifications were made in order to extend the domain of applicability of the model from theory development to more applied questions. Sensitivity analyses with the updated model revealed a dichotomy in the epidemiological effects of fitness parameters of $P$. infestans, providing two useful reference curves with which to diagnose epidemics. The model predicted rates of focal expansion that approximated observed values in the literature and also predicted disease progress curves that closely matched observational data for a number of cultivar-isolate combinations. Thus, the model is able to translate measured resistance components, weather data, and initial conditions into realistic epidemics, confirming its utility as a tool in the analysis and diagnosis of epidemics.

Simulation experiments to improve understanding about the effect of model parameters and initial conditions on the shape of disease progress curves revealed that each separate resistance component does not provide a unique mechanism for influencing the shape of disease progress curves; the six parameters $(\delta, \gamma, \mathrm{\imath}, \lambda$, $\rho$, and $\sigma$ ) used in this study present only four alternatives for altering the shape of curves (Fig. 1). Furthermore, it is apparent that a fixed latent period for all cultivar-isolate interactions is a useful simplifying assumption as disease progress was relatively insensitive to changes in its value. Separation of the epidemic processes of lesion expansion and lesion propagation provided two useful reference curves (Fig. 2) with which to formulate hypotheses regarding observed and predicted epidemics. Using these reference curves, it would appear that observed epidemics in 2004 were dominated to a greater degree by lesion propagation, relative to epidemics in 2002 (Fig. 4). The model predicted three blight days (days that are suitable for infection) during the course of the 2002 experiments, and 7 blight days over the duration of the 2004 field experiments, therefore it seems plausible that this effect is weather related.

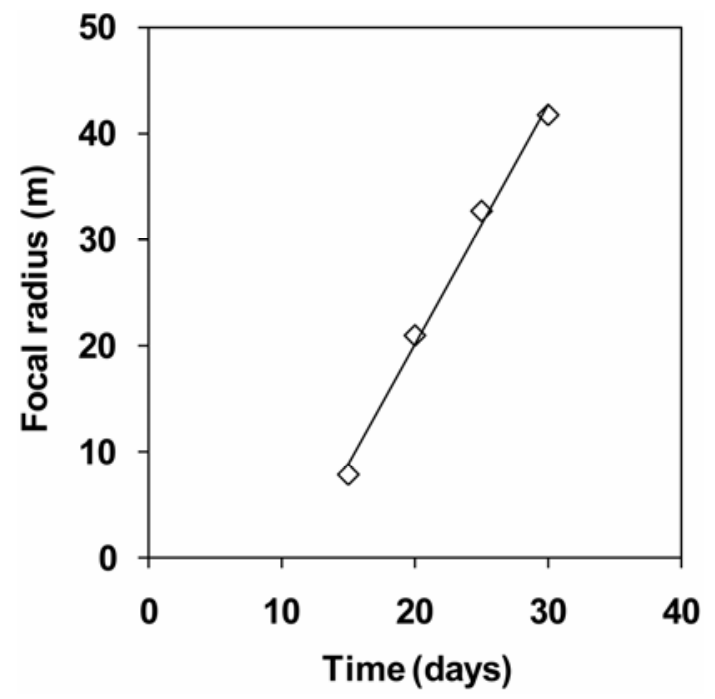

Fig. 3. Radial expansion of a simulated potato late blight focus. Parameter values for the interaction between potato cultivar Bintje and isolate IPO82001 were used (Table 4 ), with $\lambda=5$ days, $\delta=1$, and $\gamma=1$ lesion on the center plant in the field. Field size was approximately 1 ha. The solid line is a linear regression of calculated focus radius on time (ignoring the initial phase of focus build up): $y=2.3 \times-25.3, R^{2}=0.99$. 
The dispersal kernel used in the model has exponentially bounded tails and as such is said to be "thin-tailed." Integrodifference equations that use thin-tailed kernels typically possess "traveling wave" solutions, where spread occurs in a wave-like manner and a constant rate of expansion is approached (15). A second class of disease expansion has dispersive epidemic waves; i.e., a wave with an ever-increasing frontal velocity. Dispersive waves can be produced when dispersal kernels are "fat-tailed," i.e., when the kernel does not have exponentially bounded tails $(6,19)$. Mechanistic, statistical, and meteorological arguments have been proposed for both types of disease spread and it is common in plant pathology and epidemiology to fit both fat-tailed (e.g., inverse power law) and thin-tailed (e.g., negative exponential) kernels to dispersal data. With specific regard to potato

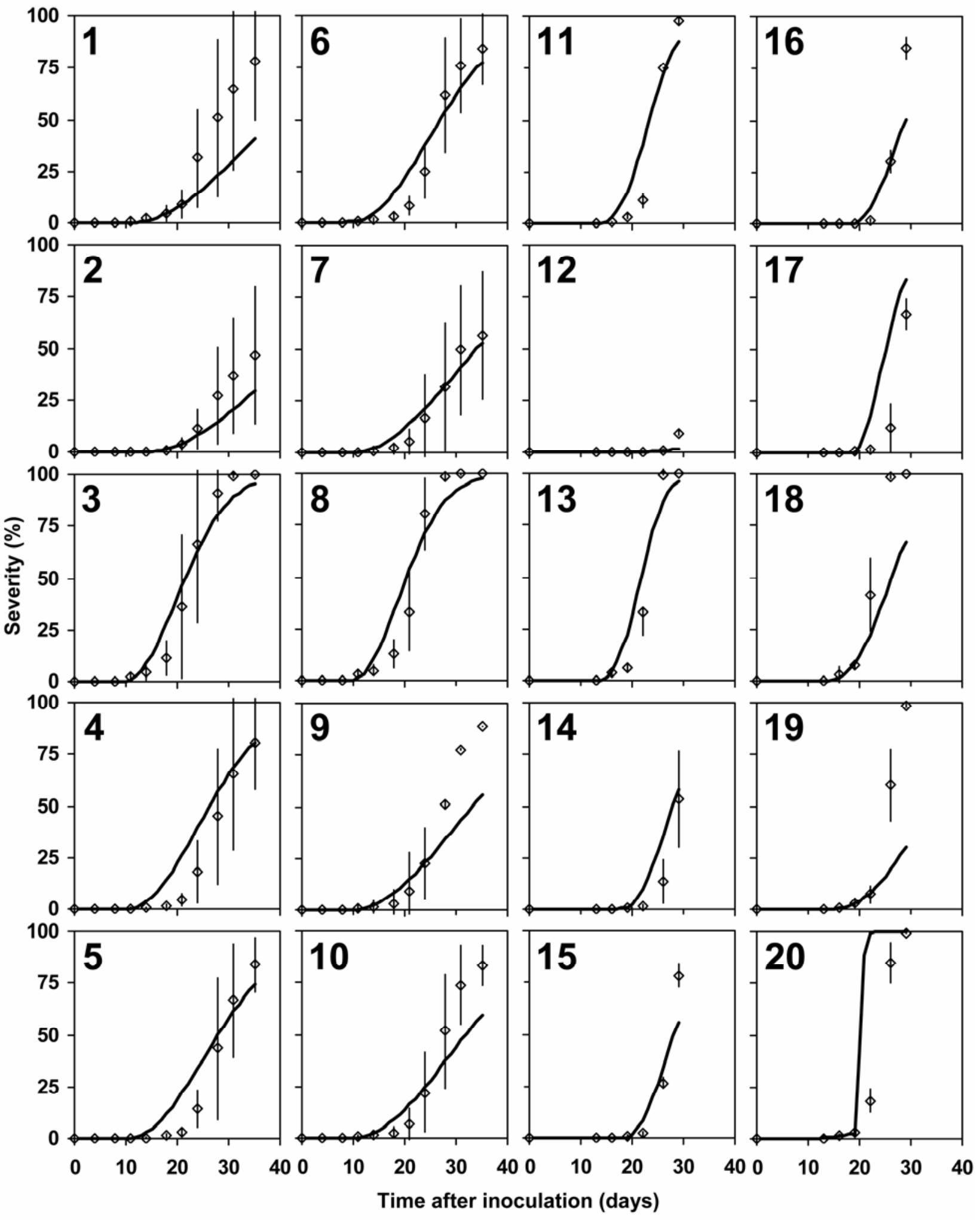

Fig. 4. Observed $(\diamond)$ and predicted $(-)$ disease progress curves of potato late blight epidemics under field conditions in the Netherlands in 2002 and 2004. Vertical lines represent the standard deviation of the observed mean blight severity. Table 2 gives a description of the isolates used to inoculate the field experiments. Table 3 provides a description of each epidemic as identified by the panel numbers. 
late blight, Minogue and Fry (20) found that spread velocity was constant over time therefore the traveling wave concept and the use of thin tailed kernels seems appropriate for the model described in this study. This observed constancy could, however, be related to the limited temporal and spatial scales of their experiments (17). Kot et al. (15) provide an excellent overview on the use of dispersal kernels to describe spatial population expansion.

Empirical data on the survival of detached sporangia reveal that most spores are killed within $1 \mathrm{~h}$ on sunny days, but many survive for several hours on cloudy days $(21,27)$. Such information suggests a wide range of possible dispersal distances. The only study in the literature that provides hard evidence of long-distance dispersal is by Zwankhuizen (36), who established that $P$. infestans sporangia from a refuse pile infected potato fields up to $900 \mathrm{~m}$ away. This suggests that it would be of relevance, and interest, to simulate potato late blight in interconnected networks of host fields. A long-distance spore dispersal and deposition submodel has recently been added to the model presented here, in addition to host growth, fungicide, and spore survival submodels, creating a spatially explicit, multiscale model of the pathosystem. It is our intention that this model will be used to identify and evaluate promising new management strategies for the regional management of potato late blight. The development and validation of the long distance spore dispersal and deposition model is described in Skelsey et al. (26).

\section{APPENDIX}

Mean absolute deviation between predicted and observed $t_{5}$ and $t_{50}$ points is given by:

$$
\mathrm{MD}=\frac{1}{\mathrm{n}} \sum_{\mathrm{i}=1}^{\mathrm{n}}\left|\mathrm{P}_{\mathrm{i}}-\mathrm{O}_{\mathrm{i}}\right|
$$

where $\mathrm{P}_{\mathrm{i}}$ and $\mathrm{O}_{\mathrm{i}}$ are the predicted and observed $t_{5}$ or $t_{50}$ points for the $i$ th epidemic.

For the following statistics, $\mathrm{P}_{\mathrm{i}}$ and $\mathrm{O}_{\mathrm{i}}$ are the predicted and observed RAUDPC for the $i$ th epidemic. The summary statistics mean prediction and mean observation, $\overline{\mathrm{P}}$ and $\overline{\mathrm{O}}$, and the standard deviations of predictions and observations, $s_{p}$ and $s_{o}$, are calculated according to formulae found in any standard statistics book. The MAE is calculated as:

$$
\text { MAE }=\frac{1}{n} \sum_{i=1}^{n}\left|P_{i}-O_{i}\right|
$$

The RMSE is given by:

$$
\mathrm{RMSE}=\left[\frac{1}{\mathrm{n}} \sum_{\mathrm{i}=1}^{\mathrm{n}}\left(\mathrm{P}_{\mathrm{i}}-\mathrm{O}_{\mathrm{i}}\right)^{2}\right]^{0.5}
$$

MAE and RMSE provide a measure of the total deviation of predicted RAUDPC from observed RAUDPC.

Ordinary least squares regression of $\mathrm{P}_{\mathrm{i}}$ on $\mathrm{O}_{\mathrm{i}}$ is used to calculate $\mathrm{RMSE}_{\mathrm{s}}$ and $\mathrm{RMSE}_{\mathrm{u}}$ as follows:

$$
\begin{aligned}
\operatorname{RMSE}_{\mathrm{s}} & =\left[\frac{1}{\mathrm{n}} \sum_{\mathrm{i}=1}^{\mathrm{n}}\left(\hat{\mathrm{P}}_{\mathrm{i}}-\mathrm{O}_{\mathrm{i}}\right)^{2}\right]^{0.5} \\
\operatorname{RMSE}_{\mathrm{u}} & =\left[\frac{1}{\mathrm{n}} \sum_{\mathrm{i}=1}^{\mathrm{n}}\left(\mathrm{P}_{\mathrm{i}}-\hat{\mathrm{P}}_{\mathrm{i}}\right)^{2}\right]^{0.5}
\end{aligned}
$$

\begin{tabular}{|c|c|c|c|c|c|c|}
\hline \multirow[b]{2}{*}{ Code $^{2}$} & \multicolumn{2}{|c|}{$t_{5}$ (days) } & \multicolumn{2}{|c|}{$t_{50}$ (days) } & \multicolumn{2}{|c|}{ RAUDPC (\%) } \\
\hline & $\mathrm{O}$ & $\mathrm{P}$ & $\mathrm{O}$ & $\mathrm{P}$ & $\mathrm{O}$ & $\mathrm{P}$ \\
\hline 1 & 18.5 & 18.3 & 23.2 & 28.8 & 20.7 & 10.4 \\
\hline 2 & 21.5 & 21.8 & 27.5 & 33.0 & 10.6 & 6.5 \\
\hline 4 & 21.1 & 14.7 & 28.7 & 26.3 & 18.1 & 25.2 \\
\hline 5 & 21.5 & 15.2 & 28.9 & 28.0 & 17.7 & 22.1 \\
\hline 6 & 19.3 & 14.3 & 26.7 & 26.9 & 22.2 & 24.4 \\
\hline 10 & 19.8 & 15.8 & 27.8 & 32.0 & 20.5 & 16.8 \\
\hline 11 & 19.7 & 16.7 & 24.5 & 23.3 & 15.9 & 19.6 \\
\hline 12 & 26.1 & 26.7 & - & - & 0.6 & 0.2 \\
\hline 13 & 17.8 & 16.1 & 23.0 & 21.8 & 22.1 & 24.5 \\
\hline 14 & 23.2 & 20.9 & 28.8 & 27.9 & 4.6 & 8.3 \\
\hline 15 & 22.5 & 21.1 & 27.4 & 28.2 & 7.6 & 7.8 \\
\hline
\end{tabular}

where $\hat{\mathrm{P}}_{\mathrm{i}}=\mathrm{a}+\mathrm{bO}_{\mathrm{i}}$, and $\mathrm{a}=\mathrm{y}$-intercept of the linear regression and $\mathrm{b}=$ slope of the linear regression. The $\mathrm{RMSE}_{\mathrm{s}}$ is a measure of the

TABLE 5. Observed $(\mathrm{O})$ and predicted $(\mathrm{P}) t_{5}$ and $t_{50}$ values (time until 5 and $50 \%$ severity, respectively), and relative area under the disease progress curve (RAUDPC, \%) values for 20 potato late blight epidemics in the Netherlands

\begin{tabular}{|c|c|c|c|c|c|c|c|c|c|c|c|c|c|}
\hline \multicolumn{6}{|c|}{ Summary univariate statistics ${ }^{\mathrm{x}}$} & \multicolumn{3}{|c|}{ OLS coefficients $^{\mathrm{y}}$} & \multicolumn{5}{|c|}{ Difference measures ${ }^{\mathrm{z}}$} \\
\hline $\mathrm{MD}_{5}$ & $\mathrm{MD}_{50}$ & $\overline{\mathrm{O}}$ & $\overline{\mathrm{P}}$ & $\mathrm{s}_{\mathrm{O}}$ & $\mathrm{s}_{\mathrm{p}}$ & $\mathrm{n}$ & $\mathrm{a}$ & $\mathrm{b}$ & MAE & RMSE & $\mathrm{RMSE}_{\mathrm{s}}$ & $\mathrm{RMSE}_{\mathrm{u}}$ & $R_{\text {pred }}^{2}$ \\
\hline 2.3 & 2.7 & 16.9 & 17.1 & 9.6 & 10.7 & 20.0 & 0.9 & 1.5 & 4.6 & 5.9 & 0.7 & 5.8 & 0.6 \\
\hline
\end{tabular}

${ }^{\mathrm{z}}$ Code used to identify the epidemic. Epidemics are described in Table 3. The same code is used in Figure 4. In epidemics 1, 2, and 19, predicted disease progress curves failed to reach $50 \%$ disease severity therefore observed and predicted $t_{25}$ values (time until $25 \%$ severity is reached) are given for comparison. In epidemic 12 , predicted and observed disease was limited and $t_{1}$ values (time until $1 \%$ severity is reached) are given for comparison.

TABLE 6. Quantitative measures of model performance

${ }^{\mathrm{x}}$ Mean absolute deviation, MD (days), calculated using equation $\mathrm{A} 1$ in the appendix. $\mathrm{MD}_{5}$ and $\mathrm{MD}_{50}$ give the mean deviation between observed and predicted $t_{5}$ and $t_{50}$ values respectively (time until 5 and $50 \%$ severity). $\overline{\mathrm{O}}$ and $\overline{\mathrm{P}}(\%)$ are the mean observed and predicted RAUDPC $(\%)$, and $\mathrm{s}_{\mathrm{o}}$ and $\mathrm{s}_{\mathrm{p}}(\%)$ are the standard deviations for observed and predicted RAUDPC. Observed and predicted $t_{5}$ and $t_{50}$ values and RAUDPC are given in Table 5.

y Ordinary least squares regression of predicted on observed RAUDPC, where $\mathrm{a}=\mathrm{y}$-intercept of the linear regression $(\%)$ and $\mathrm{b}=$ slope of the linear regression $(-)$.

${ }^{\mathrm{z}}$ Mean absolute error, MAE (\%); root mean square error, RMSE (\%); systematic root mean square error, RMSE $(\%)$; unsystematic root mean square error, $\operatorname{RMSE}_{\mathrm{u}}(\%)$; and Turchin's coefficient of prediction $(28), R_{\text {pred }}^{2}(-)$, calculated on observed and predicted RAUDPC using equations A2 to A6 in the appendix. 
deviation of the linear regression line from the one to one line and therefore indicates systematic bias. The $\mathrm{RMSE}_{\mathrm{u}}$ is a measure of the random deviation around the regression line. Since the system is conservative for the squared values of these measures (i.e., $\mathrm{RMSE}^{2}=\mathrm{RMSE}_{\mathrm{s}}^{2}+\mathrm{RMSE}_{\mathrm{u}}^{2}$ ), they can be used to calculate the proportions of the RMSE arising from systematic and unsystematic errors.

Turchin's coefficient of prediction, $R_{\text {pred }}^{2}(-)$, is used to assess overall model performance (29):

$$
R_{\text {pred }}^{2}=1-\frac{\sum_{\mathrm{i}=1}^{\mathrm{n}}\left(\mathrm{P}_{\mathrm{i}}-\mathrm{O}_{\mathrm{i}}\right)^{2}}{\sum_{\mathrm{i}=1}^{\mathrm{n}}\left(\overline{\mathrm{O}}-\mathrm{O}_{\mathrm{i}}\right)^{2}}
$$

$R_{\text {pred }}^{2}$ describes how much better a model does compared with using the mean observation as a simple forecaster. The closer $R_{\text {pred }}^{2}$ is to one, the higher the accuracy of the prediction.

\section{ACKNOWLEDGMENTS}

Funding for this study was provided by the Dutch Ministry of Agriculture, Nature Management, and Fisheries through the Umbrella Plan Phytophthora (DWK 427). We thank W. Flier, T. van den Bosch, P. van Bekkum, and $\mathrm{H}$. van Raaij from PRI for their various roles in planning and executing the field and laboratory experiments. Thanks also to J. Withagen from PRI for his help in all matters statistical.

\section{LITERATURE CITED}

1. Aylor, D. E. 1999. Biophysical scaling and the passive dispersal of fungal spores: Relationship to integrated pest management strategies. Agric. Forest Meteorol. 97:275-292.

2. Andrade-Piedra, J. L., Hijmans, R. J., Juarez, H. S., Forbes, G. A., Shtienberg, D., and Fry, W. E. 2005. Simulation of potato late blight in the Andes. II: Validation of the LATEBLIGHT model. Phytopathology 95:1200-1208.

3. Black, W., Mastenbroek, C., Mills, W. R., and Peterson, L. C. 1953. A proposal for an international nomenclature of races of Phytophthora infestans and of genes controlling immunity in Solanum demissum derivates. Euphytica 2:173-240.

4. Campbell, C. L., and Madden, L. V. 1990. Introduction to Plant Disease Epidemiology. John Wiley \& Sons, New York.

5. de Jong, M. D., Bourdôt, G. W., Powell, J., and Goudriaan, J. 2002. A model of the escape of Sclerotinia sclerotiorum ascospores from pasture. Ecol. Model. 150:83-105.

6. Ferrandino, F. J. 1993. Dispersive epidemic waves: I. focus expansion within a linear planting. Phytopathology 83:795-802.

7. Ferrandino, F. J., and Aylor, D. E. 1985. An explicit equation for deposition velocity. Bound-Lay. Meteorol. 31:197-201.

8. Flier, W. G., and Turkensteen, L. J. 1999. Foliar aggressiveness of Phytophthora infestans in three potato growing regions in the Netherlands. Eur. J. Plant Pathol. 105:381-388.

9. Forster, M., and Sober, E. 1994. How to tell when simpler, more unified, or less ad-hoc theories will provide more accurate predictions. Brit. J. Philos. Sci. 45:1-35.

10. Gregory, P. H. 1973. Page 377 in: The Microbiology of the Atmosphere. Wiley, New York.

11. Goedhart, P. W., and Thissen, J. T. N. M. 2006. Biometris Genstat Procedure Library Manual, 9th ed. PRI Biometris, the Netherlands.

12. Hirst, J. M. 1953. Changes in atmospheric spore content: Diurnal periodicity and the effects of weather. Trans. Br. Mycol. Soc. 36:375-393.

13. Hyre, R. A. 1950. Spore traps as an aid in forecasting several downy mildew type diseases. Plant Dis. Rep. 190(suppl.):S14-18.

14. James, C. 1971. A manual of assessment keys for plant diseases. Canada
Department of Agriculture. Pub. No. 1458.

15. Kot, M., Lewis, M. A., and van den Driessche, P. 1996. Dispersal data and the spread of invading organisms. Ecology 77:2027-2042.

16. Lannou, C., de Vallavieille-Pope, C., and Goyeau, H. 1994. Host mixture efficacy in disease control: Effects of lesion growth analyzed through computer-simulated epidemics. Plant Pathol. 43:651-662.

17. Madden, L. V., Hughes, G., and van den Bosch, F. 2007. The Study of Plant Disease Epidemics. The American Phytopathological Society, St. Paul, MN.

18. Malcolmson, J. F., and Black, W. 1966. New R genes in Solanum demissum Lindl. and their complementary races of Phytophthora infestans (Mont.) de Bary. Euphytica 15:199-203.

19. Minogue, K. P. 1989. Diffusion and spatial probability models for disease spread. Pages 127-143 in: Spatial Components of Plant Disease Epidemics. M. J. Jeger, ed. Prentice Hall, Englewood Cliff, NJ.

20. Minogue, K. P., and Fry, W. E. 1983. Models for the spread of plant disease: Some experimental results. Phytopathology 73:1173-1176.

21. Mizubuti, E. S. G., Aylor, D. E., and Fry, W. E. 2000. Survival of Phytophthora infestans sporangia exposed to solar radiation. Phytopathology 90:78-84.

22. Neubert, M. G., Kot, M., and Lewis, M. A. 1995. Dispersal and pattern formation in a discrete-time predator-prey model. Theor. Popul. Biol. 48:7-43.

23. Paysour, R. E., and Fry, W. E. 1983. Interplot interference: A model for planning field experiments with aerially disseminated pathogens. Phytopathology 73:1014-1020.

24. Rotem, J., Cohen, Y., and Putter, J. 1970. Relativity of limiting and optimum inoculum loads, wetting durations, and temperatures for infection by Phytophthora infestans. Phytopathology 61:275-278.

25. Skelsey, P., Rossing, W. A. H., Kessel, G. J. T., Powell, J., and van der Werf, W. 2005. Influence of host diversity on development of epidemics: An evaluation and elaboration of mixture theory. Phytopathology 95:328338.

26. Skelsey, P., van der Werf, W., and Holtslag, A. A. M. 2008. Development and validation of a quasi-Gaussian plume model for the transport of botanical spores. Agric. Forest Meterol. 148:1383-1394.

27. Sunseri, M. A., Johnson, D. A., and Dasgupta, N. 2002. Survival of detached sporangia of Phytophthora infestans exposed to ambient, relatively dry atmospheric conditions. Am. J. Potato Res. 79:443-450.

28. Swallow, W. H. 1987. Relative mean squared error and cost considerations in choosing group size for group testing to estimate infection rates and probabilities of transmission. Phytopathology 77:1376-1381.

29. Turchin, P. 2003. Complex Population Dynamics: A Theoretical/ Empirical Synthesis. Princeton University Press, NJ.

30. van der Plank, J. E. 1963. Plant Diseases: Epidemics and Control. Academic Press, New York

31. van der Zaag, D. E. 1956. Overwintering en epidemiologie van Phytophthora infestans, tevens enige nieuwe bestrijdingsmogelijkheden. Ph.D. thesis. Landbouwhogeschool, Wageningen.

32. van Oijen, M. 1992. Evaluation of breeding strategies for resistance and tolerance to late blight in potato by means of simulation modeling. Neth. J. Plant Pathol. 98:3-11.

33. van Oijen, M. 1992. Selection and use of a mathematical model to evaluate components of resistance to Phytophthora infestans in potato. Eur. J. Plant Pathol. 98:192-202.

34. Willmott, C. J. 1981. On the validation of models. Phys. Geogr. 2:184194.

35. Willmott, C. J., Ackleson, S. G., Davis, R. E., Feddema, J. J., Klink, K. M., Legates, D. R., O’Donnell, J., and Rowe, C. M. 1985. Statistics for the evaluation and comparison of models. J. Geophys. Res. 90:8995-9005.

36. Zwankhuizen, M. J. 1998. Development of potato late blight epidemics: Disease foci, disease gradients, and infection sources. Phytopathology 88:754-763.

37. Zwankhuizen, M. J., and Zadoks, J. C. 2002. Phytophthora infestans's 10year truce with Holland: A long-term analysis of potato late-blight epidemics in the Netherlands. Plant Pathol. 51:413-423. 